\title{
FAMILIES OF NONNEGATIVE DIVISORS
}

\author{
BY \\ THEODORE J. BARTH
}

Introduction. This paper presents a generalization to $n$ dimensions of some results which Oka [8] obtained in the space of 2 complex variables. The more modern (and slightly more general) terminology of divisors replaces Oka's "surfaces caractéristiques". Let $G$ be a complex manifold with structure sheaf $\mathfrak{D}$. For $z \in G$, let $\mathfrak{D}_{z}$ be the stalk of $\mathfrak{D}$ over $z$ and $\mathfrak{w}_{z}$ be the maximal ideal in $\mathfrak{D}_{z}$. If $f$ is a holomorphic function on the open subset $U$ of $G$ and $z \in U$, we define

$$
\nu_{f}(z)=\sup \left\{j \mid f_{z} \in \mathfrak{w}_{z}^{j}\right\} \leqq \infty,
$$

where $f_{z}$ is the germ of $f$ at $z$. A function $\nu$, defined on $G$, is said to be a nonnegative divisor iff each $a \in G$ possesses an open connected neighborhood $U$ on which there is a holomorphic function $f \not \equiv$ with $\nu_{f}=\nu \mid U$. The volume $V_{v}(U)$ of a divisor $\nu$ in an open set $U$ is defined in $\S 4$. The principal result of this paper is the estimate on volumes (9.1) which is prestated below:

LEMMA. Let $B(r)$ denote the open ball of radius $r$ in $C^{n-1}$, and let $D(r)$ denote the open disk of radius $r$ in $C$. Let $0<R_{0}<R_{1}<R, 0<R^{\prime \prime}<R_{1}^{\prime}<R^{\prime}$, and define

$$
\begin{aligned}
& \Omega=B(R) \times D\left(R^{\prime}\right), \quad \Omega_{1}=B\left(R_{1}\right) \times D\left(R_{1}^{\prime}\right), \\
& \Gamma=B\left(R_{0}\right) \times D\left(R^{\prime}\right) \cup B(R) \times\left(D\left(R^{\prime}\right)-\left[D\left(R^{\prime \prime}\right)\right]^{-}\right) .
\end{aligned}
$$

Then there exists a constant $C=C\left(n ; R_{0}, R_{1}, R ; R^{\prime \prime}, R_{1}^{\prime}, R^{\prime}\right)$ such that $V_{v}\left(\Omega_{1}\right)$ $\leqq C \cdot V_{v}(\Gamma)$ for every nonnegative divisor $\nu$ on $\Omega$.

In 1962, Oka [8] furnished a proof for the case $n=2$. His proof depends on the construction of a principal function to a given divisor, and this construction does not generalize to higher dimensions. Recently O. Fujita [4], using polycylinders instead of balls, has obtained a result which is, for practical purposes, equivalent to the above lemma. Fujita proceeds by induction on $n$, starting with [8] for the case $n=2$; only the induction step from $n=2$ to $n=3$ is actually carried out. Since [8] is rather difficult to read, a proof independent of it (such as the one in this paper) seems to be worthwhile.

We prove the lemma by applying the elementary wiggling methods of $\S 8$ to an apparently weaker lemma (7.1). The material appearing before $\$ 7$ is entirely devoted to the proof of 7.1. In $\$ 10$ we verify a folk theorem which enables us to obtain the applications in $\S 11$ and $\S 12$ :

Application 1. Let $\mathfrak{R}=\left\{\nu_{\lambda}\right\}_{\lambda \in \Lambda}$ be a family of nonnegative divisors on the Stein

Received by the editors January 9, 1967. 
manifold $G$, and let $A=\left\{a_{\lambda}\right\}_{\lambda \in \Lambda}$ be a family of nonnegative real numbers. Then the set $D$ of points of the first kind (with respect to $\mathfrak{N}$ and $A$ ) is a Stein manifold.

APPLICATION 2. Let $\mathfrak{N}$ be a family of nonnegative divisors on the Stein manifold $G$. Then the normality domain of $\mathfrak{R}$ is a Stein manifold.

Application 1 was proved first by Oka [8] in the case of 2 variables and recently by O. Fujita [4] in the general case. In 1934, Oka [7] indicated Application 2 for the case of 2 variables; O. Fujita [3] has given a complete direct proof; we obtain Application 2 as an immediate corollary of Application 1 and the Montel theorem of Stoll [13].

This paper is essentially the author's doctoral dissertation, written during the tenure of a National Science Foundation graduate fellowship at the University of Notre Dame. The author wishes to take this opportunity to thank his thesis director, Professor Wilhelm Stoll, for his valued assistance and advice.

1. Fubini's theorems. By a manifold we mean a pure dimensional manifold having a countable base. First we assemble a few well-known facts about differential forms, beginning with the trivial

Proposition 1.1. Let $M$ and $N$ be manifolds of class $C^{1}$, and let $\sigma: M \rightarrow N$ be a map of class $C^{1}$. Let $\chi$ be a differential form of degree $k$ on $N$. Suppose that do has rank $s<k$ at $x \in M$. Then $\sigma^{*}(\chi)(x)=0$.

A differential form of degree $m$ on a manifold of dimension $m$ is called a density. The real densities on an oriented manifold are partially ordered in the natural way. We say that the density $\phi$ is nonnegative iff $\phi \geqq 0$. For our purposes, a convenient form of Fubini's theorem is

Proposition 1.2. Let $M$ and $N$ be oriented manifolds of class $C^{1}$. Let $\phi$ and $\psi$ be nonnegative measurable densities on $M$ and $N$ respectively. Let $\pi_{1}: M \times N \rightarrow M$ and $\pi_{2}: M \times N \rightarrow N$ be the projections. Then $\pi_{1}^{*}(\phi) \wedge \pi_{2}^{*}(\psi)$ is a nonnegative measurable density on $M \times N$, and for each nonnegative measurable function fon $M \times N$,

$$
\int_{M \times N} f \pi_{1}^{*}(\phi) \wedge \pi_{2}^{*}(\psi)=\int_{y \in N}\left(\int_{M} f(\cdot, y) \phi\right) \psi(y) .
$$

Proofs of Fubini's theorem have been published by Chevalley [2, pp. 165-167] and Stoll [10, Satz 7, pp. 131-134]. In the same vein, we require [2, pp. 164-165], [10, Satz 6, pp. 128-130]:

Proposition 1.3. Let $M$ and $N$ be oriented manifolds of class $C^{1}$ and dimension $m$. Let $\sigma: M \rightarrow N$ be a local diffeomorphism of class $C^{1}$. Let $\chi$ be a nonnegative measurable density on $N$, and let $f$ be a nonnegative measurable function on $M$. Then

$$
\int_{M} f\left|\sigma^{*}(\chi)\right|=\int_{y \in N} \sum_{x \in \sigma^{-1}(y)} f(x) \chi(y) .
$$


We appeal to the famous theorem of Sard [9] in modifying 1.3 to obtain a more usable

Proposition 1.4. Let $M$ and $N$ be oriented manifolds of class $C^{\infty}$ and dimension $m$. Let $\sigma: M \rightarrow N$ be a map of class $C^{\infty}$. Let $\chi$ be a nonnegative measurable density on $N$, and let $f$ be a nonnegative measurable function on $M$. Then

$$
\int_{M} f\left|\sigma^{*}(x)\right|=\int_{y \in N} \sum_{x \in \sigma^{-1}(y)} f(x) \chi(y) .
$$

Proof. Define $M_{0}=\{x \mid x \in M, d \sigma$ has rank $m$ at $x\}$. Then $M_{0}$ is open and $\tau=\sigma \mid M_{0}: M_{0} \rightarrow N$ is a local diffeomorphism. According to 1.1 , if $x \in M-M_{0}$, then $\sigma^{*}(x)(x)=0$; hence

$$
\int_{M} f\left|\sigma^{*}(\chi)\right|=\int_{M_{0}} f\left|\sigma^{*}(\chi)\right|
$$

By 1.3,

$$
\int_{M_{0}} f\left|\sigma^{*}(\chi)\right|=\int_{y \in N} \sum_{x \in \tau^{-1}(y)} f(x) \chi(y)
$$

By Sard's theorem,

$$
\begin{aligned}
N^{\prime} & =\{y \mid y \in N, y \text { is a critical value of } \sigma\} \\
& =\left\{y \mid y \in N, \tau^{-1}(y) \neq \sigma^{-1}(y)\right\}
\end{aligned}
$$

has measure zero in $N$. Therefore

$$
\int_{y \in N} \sum_{x \in \tau^{-1}(y)} f(x) \chi(y)=\int_{y \in N} \sum_{x \in \sigma^{-1}(y)} f(x) \chi(y), \quad \text { Q.E.D. }
$$

2. The fibered divisor. In the following, $\boldsymbol{C}$ denotes the field of complex numbers; $R$, the reals; $N$, the natural numbers; $N_{0}=N \cup\{0\}$. We adopt the convention $0 \cdot \infty=\infty \cdot 0=0$.

Let $G$ be a complex manifold with structure sheaf $\mathfrak{O}$. For $z \in G$, let $\mathfrak{D}_{z}$ be the stalk of $\mathfrak{D}$ over $z$ and $\mathfrak{w}_{z}$ be the maximal ideal in $\mathfrak{D}_{z}$. If $f$ is a holomorphic function on the open subset $U$ of $G$ and $z \in U$, we define

$$
\nu_{f}(z)=\sup \left\{j \mid j \in N_{0}, f_{z} \in \mathfrak{w}_{z}^{j}\right\} \in N_{0} \cup\{\infty\},
$$

where $f_{z}$ is the germ of $f$ at $z$. A function $\nu: G \rightarrow N_{0}$ is said to be a nonnegative divisor iff each $a \in G$ possesses an open neighborhood $U$ on which there is a holomorphic function $f$ with $\nu_{f}=\nu \mid U$. The set

$$
\mathfrak{M}(\nu)=\{z \mid z \in G, \nu(z)>0\}
$$

is called the support of $\nu$; it is empty or an analytic set of pure codimension 1 . Let $\mathfrak{R}(\mathfrak{M}(\nu)$ ) denote the set of regular (simple) points of $\mathfrak{M}(\nu)$. The function $\nu$ is constant on each connectivity component of $\mathfrak{R}(\mathfrak{M}(\nu))$. 
Let $G$ and $H$ be complex manifolds, and let $\alpha: G \rightarrow H$ be a holomorphic map. Let $\nu$ be a nonnegative divisor on $H$. Let $V$ be an open connected subset of $H$ on which there is a holomorphic function $f$ with $\nu_{f}=\nu \mid V$. Define

$$
\alpha^{*}(\nu)(z)=v_{f \circ \alpha}(z) \quad \text { for } z \in \alpha^{-1}(V) .
$$

The value of $\alpha^{*}(v)(z)$ does not depend on the choice of $V$ and $f$, so that $\alpha^{*}(v): G$ $\rightarrow N_{0} \cup\{\infty\}$ is well defined. If $\alpha(V) \notin \mathfrak{M}(\nu)$ for every nonempty open subset $V$ of $G$, then $\alpha^{*}(\nu)$ is a nonnegative divisor on $G$, called the induced divisor.

Let $G$ and $H$ be complex manifolds of dimensions $m$ and $n$ respectively, and let $\rho: G \rightarrow H$ be a holomorphic map. Suppose that $d \rho$ has complex rank $n$ at each point of $G$. (This implies that $n \leqq m$.) Then, for each $z \in H, L_{z}=\rho^{-1}(z)$ is an $(m-n)$-dimensional smooth complex submanifold of $G$. Let $\iota_{z}: L_{z} \rightarrow G$ be the inclusion. If $\nu$ is a nonnegative divisor on $G$, the fibered divisor

$$
\nu_{\rho}: G \rightarrow N_{0} \cup\{\infty\}
$$

is defined by $\nu_{\rho}(w)=\iota_{\rho(w)}^{*}(v)(w)$, i.e., it is the induced divisor on each fiber of $\rho$.

LEMMA 2.1. Let $G$ and $H$ be complex manifolds of dimensions $n$ and $n-1$ respectively, and let $\rho: G \rightarrow H$ be a holomorphic map. Suppose that $d \rho$ has complex rank $n-1$ at each point of $G$. Let $\nu$ be a nonnegative divisor on $G$, and let $\chi$ be a density on $H$. Define $M=\Re(M(v))$, and let $\iota: M \rightarrow G$ be the inclusion. Then

(1) $\nu(a)=\nu_{\rho}(a)$ for each $a \in M$ such that $d(\rho \circ \iota)$ has complex rank $n-1$ at $a$;

(2) $\nu(\rho \circ \iota)^{*}(\chi)=v_{\rho}(\rho \circ \iota)^{*}(\chi)$ on $M$.

Proof. Our convention that $\infty \cdot 0=0$, applied to 1.1 , shows that it will suffice to prove (1). Since (1) is a local statement, we may assume that

(a) $G$ is an open connected neighborhood of $a=0$ in $C^{n}$;

(b) $\boldsymbol{H}$ is an open connected neighborhood of 0 in $\boldsymbol{C}^{n-1}$;

(c) $\rho\left(w_{1}, \ldots, w_{n}\right)=\left(w_{1}, \ldots, w_{n-1}\right)$ for $\left(w_{1}, \ldots, w_{n}\right) \in G$;

(d) $\rho(G)=H, \mathfrak{M}(\nu)=M$.

Since $d(\rho \circ \iota)$ has complex rank $n-1$ at $a$, we may even assume that

(e) $\rho \circ \imath: M \rightarrow H$ is biholomorphic.

Let $j=v(a)$. According to (b), $H$ is connected; therefore $M$ is connected and

$$
\begin{aligned}
\nu(w) & =j & & \text { for } w \in M, \\
& =0 & & \text { for } w \in G-M .
\end{aligned}
$$

Let $\gamma: H \rightarrow M$ be the inverse of $\rho \circ \iota$. Then

$$
\gamma\left(z_{1}, \ldots, z_{n-1}\right)=\left(z_{1}, \ldots, z_{n-1}, h\left(z_{1}, \ldots, z_{n-1}\right)\right),
$$

where $h$ is a holomorphic function on $H$ with $h(0, \ldots, 0)=0$. Define $g: G \rightarrow C$ by

$$
g\left(w_{1}, \ldots, w_{n}\right)=w_{n}-h\left(w_{1}, \ldots, w_{n-1}\right) .
$$

Then $M=\{w \mid w \in G, g(w)=0\}$. If $c=\left(c_{1}, \ldots, c_{n}\right) \in M$, then

$$
0=g(c)=c_{n}-h\left(c_{1}, \ldots, c_{n-1}\right) \text {, }
$$


and in a neighborhood of $c$,

$$
g\left(w_{1}, \ldots, w_{n}\right)=\left(w_{n}-c_{n}\right)+\left(h\left(c_{1}, \ldots, c_{n-1}\right)-h\left(w_{1}, \ldots, w_{n-1}\right)\right) ;
$$

hence $\nu_{g}(c)=1$. Therefore

$$
\begin{aligned}
\nu_{g}(w)=1 & \text { for } w \in M, \\
=0 & \text { for } w \in G-M,
\end{aligned}
$$

i.e., $\nu=\nu_{g}$ s. Define

$$
L_{0}=\rho^{-1}(0)=\left\{\left(0, \ldots, 0, w_{n}\right) \mid\left(0, \ldots, 0, w_{n}\right) \in G\right\},
$$

and let $\iota_{0}: L_{0} \rightarrow G$ be the inclusion. Then $\nu_{\rho}(a)=\iota_{0}^{*}(\nu)(0)=\iota_{0}^{*}\left(\nu_{g}^{s}\right)(0)$, and

$$
\left(g^{j} \circ \iota_{0}\right)\left(0, \ldots, 0, w_{n}\right)=g^{j}\left(0, \ldots, 0, w_{n}\right)=w_{n}^{j} .
$$

Hence $\nu_{\rho}(a)=\iota_{0}^{*}\left(\nu_{g}\right)(0)=j=v(a)$, Q.E.D.

If $M$ is a $k$-dimensional complex submanifold of the complex manifold $G, f$ is a function defined on $M, \chi$ is a differential form of degree $2 k$ on $G$, and $\iota: M \rightarrow G$ is the inclusion, we define

$$
\int_{M} f \chi=\int_{M} f_{i}^{*}(\chi)
$$

provided this integral exists.

Proposition 2.2. Let $G$ and $H$ be complex manifolds of dimensions $n$ and $n-1$ respectively, and let $\rho: G \rightarrow H$ be a holomorphic map. Suppose that $d \rho$ has complex rank $n-1$ at each point of $G$. Let $\nu$ be a nonnegative divisor on $G$, and let $f$ be $a$ measurable function defined on $M=\mathfrak{R}(\mathfrak{M}(\nu))$. Let $\chi$ be a nonnegative measurable density on $H$. Then

$$
\int_{M} v f \rho^{*}(\chi)=\int_{z \in H} \sum_{w \in \rho^{-1}(z)} v_{\rho}(w) f(w) \chi(z)
$$

provided the left integral exists.

Proof. Without loss of generality we may assume that $f \geqq 0$. Let $\iota: M \rightarrow G$ be the inclusion. By 2.1,

$$
\int_{M} \nu f \rho^{*}(\chi)=\int_{M} \nu f(\rho \circ \iota)^{*}(\chi)=\int_{M} \nu_{\rho} f(\rho \circ \iota)^{*}(\chi) .
$$

Since $\chi$ is nonnegative and $\tau=\rho \circ \iota$ is holomorphic, $\tau^{*}(\chi)$ is nonnegative. Hence, according to 1.4 ,

$$
\int_{M} \nu_{\rho} f(\rho \circ \iota)^{*}(\chi)=\int_{M} \nu_{\rho} f\left|\tau^{*}(\chi)\right|=\int_{z \in H} \sum_{w \in \tau^{-1}(z)} v_{\rho}(w) f(w) \chi(z) .
$$

Since $\rho(\mathfrak{M}(\nu)-M)$ has measure zero in $H$ and $\nu_{\rho}(w)=0$ if $w \notin \mathfrak{M}(v)$, we have

$$
\int_{z \in H} \sum_{w \in \tau^{-1}(z)} \nu_{\rho}(w) f(w) \chi(z)=\int_{z \in H} \sum_{w \in \rho^{-1}(z)} \nu_{\rho}(w) f(w) \chi(z), \quad \text { Q.E.D. }
$$


By using some results of Stoll [12, Hilfssatz 6.3 and Hilfssatz 6.4, pp. 396-397], 2.2 can be proved without Sard's theorem.

3. Differential forms in a unitary space. If $G$ is a complex manifold and $\phi$ is a differential form of class $C^{1}$ on $G$, the exterior derivative $d \phi$ is defined. We have $d=\partial+\bar{\partial}$, where $\partial$ and $\bar{\partial}$ are bihomogeneous operators of bidegrees $(1,0)$ and $(0,1)$ respectively. Define $d^{\perp}=i(\partial-\bar{\partial})$.

A unitary space is a complex vector space on which a positive definite Hermitian form (inner product) is defined. In the following, all unitary spaces are assumed finite dimensional, and $\boldsymbol{C}^{n}$ is considered as a unitary space under the ordinary (complex) inner product.

Let $Z$ be a $k$-dimensional unitary space with inner product (|). If $z \in Z$, the length of $z$ is $\psi(z)=|z|=(z \mid z)^{1 / 2}$. On $Z$ we define the differential forms

$$
\begin{aligned}
(d z \mid z) & =\partial(z \mid z), \quad(z \mid d z)=\bar{\partial}(z \mid z), \\
(d z \mid d z) & =\partial \bar{\partial}(z \mid z)=-d(d z \mid z)=d(z \mid d z), \\
v(z) & =\frac{1}{2} i(d z \mid d z)=\frac{1}{2} i \partial \bar{\partial}(z \mid z), \\
v_{q} & =(1 / q !) v \wedge \cdots \wedge v(q \text {-times }), \quad q=1, \ldots, k, \\
\eta(z) & =\frac{1}{4} i[(z \mid d z)-(d z \mid z)] .
\end{aligned}
$$

On $Z-\{0\}$ we define the differential forms

$$
\begin{aligned}
\omega(z) & =\frac{1}{2} i|z|^{-4}\left[|z|^{2}(d z \mid d z)-(d z \mid z) \wedge(z \mid d z)\right], \\
\omega_{q} & =(1 / q !) \omega \wedge \cdots \wedge \omega(q \text {-times }), \quad q=1, \ldots, k .
\end{aligned}
$$

The euclidean volume element of $Z$ is $v_{k}$. We have

$$
v_{k}=d^{\perp} \psi \wedge d \psi \wedge v_{k-1}=2 i \partial \psi \wedge \bar{\partial} \psi \wedge v_{k-1}
$$

Define $B=\{z|z \in Z| z \mid,<1\}, S=\{z|z \in Z| z \mid,=1\}$. Orient $S$ towards the exterior of $B$, let $\sigma$ be the euclidean volume element of $S$, and let $\iota: S \rightarrow Z$ be the inclusion. Then

$$
\sigma=-\imath^{*}\left(d^{\perp} \psi \wedge v_{k-1}\right)
$$

Define $\theta: Z-\{0\} \rightarrow S$ by $\theta(z)=z /|z|$. Then $(\iota \circ \theta)^{*}(v)=\omega[10$, p. 142].

When we are considering more than one unitary space and confusion is possible, we will use superscripts on the above quantities to indicate the space to which they refer.

The important result of this section is the formula given in

LEMma 3.1. Let $Z$ be a $k$-dimensional unitary space. Then $\theta^{*}(\sigma)=\left(2 / \psi^{2 k}\right) \eta \wedge v_{k-1}$.

Proof. As mentioned above, we have $\sigma=-\imath^{*}\left(d^{\perp} \psi \wedge v_{k-1}\right)$. Now

$$
\begin{aligned}
-\left(d^{\perp} \psi\right)(z) & =-i(\partial-\bar{\partial})(z \mid z)^{1 / 2} \\
& =-\frac{1}{2} i|z|^{-1}[\partial(z \mid z)-\bar{\partial}(z \mid z)] \\
& =(2 / \psi(z)) \eta(z)
\end{aligned}
$$


i.e., $-d^{\perp} \psi=(2 / \psi) \eta$. Hence

$$
\begin{aligned}
\sigma & =\iota^{*}\left((2 / \psi) \eta \wedge v_{k-1}\right), \\
\theta^{*}(\sigma) & =(\iota \circ \theta)^{*}\left((2 / \psi) \eta \wedge v_{k-1}\right) \\
& =2(\iota \circ \theta)^{*}\left(\eta \wedge v_{k-1}\right) .
\end{aligned}
$$

Note that

$$
d|z|=(\partial+\bar{\partial})(z \mid z)^{1 / 2}=\frac{1}{2}|z|^{-1}[(d z \mid z)+(z \mid d z)] .
$$

Let $e_{1}, \ldots, e_{k}$ be an orthonormal basis for $Z$, and define $z_{j}: Z \rightarrow C$ by $z_{j}(z)$ $=\left(z \mid e_{j}\right), j=1, \ldots, k$. Then

$$
\begin{aligned}
(\iota \circ \theta)^{*}(z \mid d z) & =(\iota \circ \theta) *\left(\sum z_{j} d \bar{z}_{j}\right)=\sum\left(z_{j} /|z|\right) d\left(\bar{z}_{j} /|z|\right), \\
d\left(\bar{z}_{j}|| z \mid\right) & =|z|^{-2}\left[|z| d \bar{z}_{j}-\bar{z}_{j} d|z|\right] ; \\
(\iota \circ \theta)^{*}(z \mid d z) & =|z|^{-3} \sum z_{j}\left\{|z| d \bar{z}_{j}-\left.\bar{z}_{j}\left|\frac{1}{2}\right| z\right|^{-1}[(z \mid d z)+(d z \mid z)]\right\} \\
& =|z|^{-3}\left\{|z|(z \mid d z)-\frac{1}{2}|z|[(z \mid d z)+(d z \mid z)]\right\} \\
& =|z|^{-2} \cdot \frac{1}{2}[(z \mid d z)-(d z \mid z)] \\
& =(2 / i)|z|^{-2} \eta(z) .
\end{aligned}
$$

Similarly,

$$
\left(\llcorner\theta)^{*}(d z \mid z)=-(2 / i)|z|^{-2} \eta(z)\right.
$$

Therefore

$$
\begin{aligned}
(\iota \circ \theta)^{*}(\eta)(z) & =(\iota \circ \theta)^{*}\left(\frac{1}{4} i[(z \mid d z)-(d z \mid z)]\right) \\
& =|z|^{-2} \eta(z)
\end{aligned}
$$

i.e., $(\iota \circ \theta)^{*}(\eta)=\left(1 / \psi^{2}\right) \eta$. Since $(\iota \circ \theta)^{*}(v)=\omega$, we have

$$
\theta^{*}(\sigma)=2(\iota \circ \theta)^{*}\left(\eta \wedge v_{k-1}\right)=\left(2 / \psi^{2}\right) \eta \wedge \omega_{k-1} .
$$

But

$$
\omega(z)=|z|^{-2} v(z)-\frac{1}{2} i|z|^{-4}(d z \mid z) \wedge(z \mid d z)
$$

and

$$
\eta(z)=\frac{1}{4} i[(z \mid d z)-(d z \mid z)]
$$

hence

$$
\eta(z) \wedge \omega_{k-1}(z)=|z|^{-(2 k-2)} \eta(z) \wedge v_{k-1}(z),
$$

i.e.,

$$
\eta \wedge \omega_{k-1}=\left(1 / \psi^{2 k-2}\right) \eta \wedge v_{k-1} .
$$

Therefore $\theta^{*}(\sigma)=\left(2 / \psi^{2 k}\right) \eta \wedge v_{k-1}$, Q.E.D. 
For reference we state the following well-known application of 1.2 (Fubini's theorem):

Proposition 3.2. Let $Z$ be a $k$-dimensional unitary space. Let $0 \leqq s<r$, and define $A=\{z|z \in Z, s<| z \mid<r\}$. Let $f$ be a nonnegative measurable function on $A$. Then

$$
\int_{A} f v_{k}=\int_{s}^{r}\left(\int_{a \in S} f(t a) \sigma(a)\right) t^{2 k-1} d t
$$

In particular, if $f\left(t_{1} a\right)=f\left(t_{2} a\right)=g(a)$ for $t_{1}, t_{2}$ with $s<t_{1}<t_{2}<r$ and all $a \in S$, then

$$
\int_{A} f v_{k}=(1 / 2 k)\left(r^{2 k}-s^{2 k}\right) \int_{S} g \sigma .
$$

4. The first estimate. In the following, $n$ will always denote an integer greater than 1 . Let $V$ be a unitary space. We say that $c \in V$ is a unit vector iff $|c|=1$. If $c$ is a unit vector, let

$$
E(c)=\{v \mid v \in V,(v \mid c)=0\}
$$

denote the space of vectors orthogonal to $c$, and let $\rho_{c}: V \rightarrow E(c)$ denote the projection defined by

$$
\rho_{c}(\boldsymbol{v})=\boldsymbol{v}-(\boldsymbol{v} \mid \boldsymbol{c}) \boldsymbol{c} .
$$

Since $E(c)$ is a vector subspace of $V$, it is a unitary space under the same inner product.

Let $V$ be an $n$-dimensional unitary space, and let $\nu$ be a nonnegative divisor on the open subset $G$ of $V$. For each open subset $U$ of $G$, the volume of $\nu$ in $U$ is (by definition)

$$
V_{v}(U)=\int_{M} v v_{n-1}
$$

where $M=\mathfrak{R}(\mathfrak{M}(\nu)) \cap U$.

Proposition 4.1. Let $c$ be a unit vector in the n-dimensional unitary space $V$, and let $Z=E(c)$. Let $\nu$ be a nonnegative divisor on the open subset $G$ of $V$. Define $M=$ $\Re(\mathfrak{M}(\nu))$ and $\rho=\rho_{c} \mid G: G \rightarrow Z$. Then

$$
\int_{M} \nu \rho^{*}\left(v_{n-1}^{Z}\right)=\int_{z \in Z} \sum_{v \in \rho^{-1}(z)} v_{\rho}(v) v_{n-1}^{Z}(z) \leqq V_{v}(G)
$$

Proof. The above equality follows immediately from 2.2 . Let $\iota_{M}: M \rightarrow G$ be the inclusion. When expressed in coordinate notation, the inequality

$$
\iota_{M}^{*}\left(\rho^{*}\left(v_{n-1}^{Z}\right)\right) \leqq \iota_{M}^{*}\left(v_{n-1}^{V}\right)
$$

is obvious. Hence

$$
\int_{M} \nu \rho^{*}\left(v_{n-1}^{Z}\right) \leqq \int_{M} \nu v_{n-1}^{v}=V_{v}(G), \quad \text { Q.E.D. }
$$


5. The second estimate. The main part of the proof of the second estimate is given in the geometrical

Lemma 5.1. Let $0<R^{\prime}<R^{\prime \prime}$. Define

$$
D^{\prime \prime}=\left\{w|| w \mid<R^{\prime \prime}\right\} \subset C, \quad I=\{t \mid 0 \leqq t \leqq 1\} \subset R .
$$

Let $F: I \times D^{\prime \prime} \rightarrow C$ be a continuous function such that $f(t, \cdot)$ is holomorphic and $\not \equiv 0$ for each $t \in I$.

(1) If $0 \notin f\left(I \times\left\{w|| w \mid=R^{\prime}\right\}\right)$, then

$$
\sum_{|w|<R^{\prime}} \nu_{f(1, \cdot)}(w)=\sum_{|w|<R^{\prime}} \nu_{f(0, \cdot)}(w) .
$$

(2) In any case,

$$
\sum_{|w|<R^{\prime}} \nu_{f(1, \cdot)}(w) \leqq \sum_{|w|<R^{\prime}} v_{f(0, \cdot)}(w)+\sum_{0 \leqq t<1} \sum_{|w|=R^{\prime}} v_{f(t, \cdot)}(w) .
$$

Proof. (1) Define $f^{\prime}: I \times D^{\prime \prime} \rightarrow C$ by $f^{\prime}(t, w)=\partial f(t, w) / \partial w$. In view of the Cauchy integral formula, $f^{\prime}$ is continuous. According to the argument principle (see any text, for example [6, Vol. I, p. 252]),

$$
a(t)=\sum_{|w|<R^{\prime}} \nu_{f(t, \cdot)}(w)=\frac{1}{2 \pi i} \int_{|\zeta|=R^{\prime}} \frac{f^{\prime}(t, \zeta)}{f(t, \zeta)} d \zeta .
$$

The function $a(t)$ is continuous and integral valued, hence constant. In particular, $a(1)=a(0)$.

(2) If

$$
\sum_{|w|=R^{\prime}} \nu_{f(t, \cdot)}(w)>0
$$

for infinitely many $t \in I$, there is nothing to prove. We may therefore assume that

$$
\sum_{|w|=R^{\prime}} \nu_{f(t, \cdot)}(w)=0 \quad \text { for } t \in I-\left\{t_{1}, \ldots, t_{k}\right\},
$$

where $0=t_{1}<t_{2}<\cdots<t_{k}=1$.

Fix $j \in N$ with $1 \leqq j \leqq k-1$. There exists $r<R^{\prime}$ such that $f\left(t_{j+1}, w\right) \neq 0$ for $r \leqq|w|<R^{\prime}$. Take $t^{\prime}$ with $t_{j}<t^{\prime}<t_{j+1}$ such that $f(t, w) \neq 0$ if $t^{\prime}<t<t_{j+1}$ and $|w|=r$. From part (1),

$$
\sum_{|w|<R^{\prime}} \nu_{f\left(t_{j+1}, \cdot\right)}(w)=\sum_{|w|<r} \nu_{f\left(t_{j+1}, \cdot\right)}(w)=\sum_{|w|<r} \nu_{f\left(t^{\prime}, \cdot\right)}(w) \leqq \sum_{|w|<R^{\prime}} \nu_{f\left(t^{\prime}, \cdot\right)}(w) .
$$

Similarly, there exists $t^{\prime \prime}$ with $t_{j}<t^{\prime \prime}<t_{j+1}$ and

$$
\sum_{|w|<R^{\prime}} \nu_{f\left(t^{\prime \prime}, \cdot\right)}(w) \leqq \sum_{|w| \leqq R^{\prime}} \nu_{f\left(t_{j}, \cdot\right)}(w) .
$$

According to (1),

$$
\sum_{|w|<R^{\prime}} \nu_{f\left(t^{\prime}, \cdot\right)}(w)=\sum_{|w|<R^{\prime}} v_{f\left(t^{\prime \prime}, \cdot\right)}(w) .
$$

Combining the last three relations, we obtain

$$
\sum_{|w|<R^{\prime}} \nu_{f\left(t_{j+1}, \cdot\right)}(w)-\sum_{|w|<R^{\prime}} \nu_{f\left(t_{j}, \cdot\right)}(w) \leqq \sum_{|w|=R^{\prime}} \nu_{f\left(t_{j}, \cdot\right)}(w)
$$

for $j=1, \ldots, k-1$. Summing over $j$ gives the desired inequality, Q.E.D. 
The idea for this second estimate was given by Oka $[8$, p. 10].

Lemma 5.2. Let $c$ be a unit vector in the unitary space $V$, and let $Z=E(c)$. Let $0<R_{0}<R, R^{\prime}>0$. Let $G$ be an open neighborhood of

$$
\left\{z+w c\left|z \in Z, R_{0} \leqq\right| z|\leqq R,| w \mid \leqq R^{\prime}\right\},
$$

and let $\nu$ be a nonnegative divisor on $G$. Define $\rho=\rho_{c} \mid G: G \rightarrow Z$, and let $z \in Z$ with $R_{0} \leqq|z| \leqq R$. Then

$$
\sum_{|w|<R^{\prime}} \nu_{\rho}(z+w \boldsymbol{c}) \leqq \sum_{|w|<R^{\prime}} \nu_{\rho}\left(R_{0} z /|z|+w \boldsymbol{c}\right)+\sum_{R_{0} \leq t \leq|z| \mid} \sum_{|w|=R^{\prime}} \nu_{\rho}(z z|| z \mid+w \boldsymbol{c}) .
$$

Proof. We simply apply 5.1 in the obvious way. Let $I=\{t \mid 0 \leqq t \leqq 1\}$, and define $\phi: I \rightarrow Z$ by

$$
\phi(t)=(1-t) R_{0} z /|z|+t z .
$$

Let $U$ be an open neighborhood of $\left\{\phi(t)+w c|t \in I| w \mid, \leqq R^{\prime}\right\}$ on which there exists a holomorphic function $g$ with $\nu_{g}=\nu \mid U$. Take $R^{\prime \prime}>R^{\prime}$ such that

$$
\left\{\phi(t)+w c|t \in I,| w \mid<R^{\prime \prime}\right\} \subset U .
$$

Define $f: I \times\left\{w|| w \mid<R^{\prime \prime}\right\} \rightarrow C$ by

$$
f(t, w)=g(\phi(t)+w c) .
$$

Then $f$ is continuous; $f(t, \cdot)$ is holomorphic for each $t \in I$; and $\nu_{f(t, .)}(w)$ $=v_{\rho}(\phi(t)+w c)$ for $0 \leqq t \leqq 1,|w|<R^{\prime \prime}$. Hence

$$
\sum_{R_{0} \leq t \leq \leq|z|} \sum_{|w|=R^{\prime}} \nu_{\rho}(t z|| z \mid+w c)=\sum_{0 \leq t \leq 1} \sum_{|w|=R^{\prime}} \nu_{f(t, .)}(w) .
$$

If $f(t, \cdot) \equiv 0$ for some $t \in I$, then

$$
\sum_{0 \leqq t \leqq 1} \sum_{|w|=R^{\prime}} \nu_{f(t, \cdot)}(w)=\infty,
$$

and the desired estimate is trivial. (Here it is necessary that the summation be over $0 \leqq t \leqq 1$, rather than $0 \leqq t<1$ as in 5.1.) If $f(t, \cdot) \not \equiv 0$ for each $t \in I$, we apply 5.1 to obtain

$$
\sum_{|w|<R^{\prime}} \nu_{f(1, \cdot)}(w) \leqq \sum_{|w|<R^{\prime}} \nu_{f(0, \cdot)}(w)+\sum_{0 \leqq t \leqq 1} \sum_{|w|=R^{\prime}} \nu_{f(t, \cdot)}(w),
$$

which is the desired estimate, Q.E.D.

6. The third estimate. Let $G$ be a complex manifold of dimension $k$, and let $x$ be a differential form of bidegree $(k-1, k-1)$ on $G$. We say that $\chi$ is semipositive definite iff for every smooth $(k-1)$-dimensional complex submanifold $M$ with inclusion $\iota_{M}: M \rightarrow G$, the induced form $\iota_{M}^{*}(\chi)$ is a nonnegative density on $M$. We give another characterization of this property in

Proposition 6.1. Let $G$ be a complex manifold of dimension $k$, and let $\chi$ be a differential form of bidegree $(k-1, k-1)$ on $G$. Then $\chi$ is semipositive definite if and 
only if for every differential form $\gamma$ of bidegree $(1,0)$ on $G$, the form $\frac{1}{2} i \gamma \wedge \bar{\gamma} \wedge \chi$ is a nonnegative density.

Proof. Let $z_{1}, \ldots, z_{k}$ be local coordinates in $G$. Then

$$
\begin{aligned}
\chi= & \left(\frac{1}{2} i\right)^{k-1} \sum_{p \neq q} a_{p q} d \bar{z}_{q} \wedge d z_{p} \wedge d z_{1} \wedge d \bar{z}_{1} \wedge \cdots\left\|^{p} \cdots\right\|^{q} \cdots \wedge d z_{k} \wedge d \bar{z}_{k} \\
& +\left(\frac{1}{2} i\right)^{k-1} \sum_{p} a_{p p} d z_{1} \wedge d \bar{z}_{1} \wedge \cdots \|^{p} \cdots \wedge d z_{k} \wedge d \bar{z}_{k},
\end{aligned}
$$

where $\|^{p}$ means that the term $d z_{p} \wedge d \bar{z}_{p}$ is omitted. The form $\chi$ is semipositive definite if and only if $\sum a_{p q} U_{p} \bar{U}_{q}$ is a semipositive definite Hermitian form [11, Satz 4.3, pp. 42-43]. If $\gamma$ is a differential form of bidegree $(1,0)$ on $G$, we have

$$
\gamma=\sum c_{j} d z_{j}, \quad \bar{\gamma}=\sum \bar{c}_{j} d \bar{z}_{j},
$$

and readily calculate

$$
\frac{1}{2} i \gamma \wedge \bar{\gamma} \wedge \chi=\left(\frac{1}{2} i\right)^{k}\left(\sum_{p, q} a_{p q} c_{q} \bar{c}_{p}\right) d z_{1} \wedge d \bar{z}_{1} \wedge \cdots \wedge d z_{k} \wedge d \bar{z}_{k}, \quad \text { Q.E.D. }
$$

We first present the third estimate in its unintegrated form:

Lemma 6.2. Let $c$ be a unit vector in the n-dimensional unitary space $V$. Define $Z=E(c)$, and let $\rho=\rho_{c}: V \rightarrow Z$. Define $S=S^{z}=\{z|z \in Z| z \mid,=1\}$, and let $\sigma=\sigma^{z}$ be the euclidean volume element of $S$. Let $r: R \rightarrow R$ be the identity function. Let $\pi_{1}: S \times R \rightarrow S, \pi_{2}: S \times R \rightarrow R$ be the projections. Define

$$
\chi=\pi_{1}^{*}(\sigma) \wedge \pi_{2}^{*}(d r)
$$

Define $w: V \rightarrow C$ by $w(v)=(v \mid c)$, and let

$$
G=\{v \mid v \in V, \rho(v) \neq 0, w(v) \neq 0\} .
$$

Define $\alpha: G \rightarrow S \times R$ by

Then

$$
\alpha(\boldsymbol{v})=(\rho(\boldsymbol{v}) /|\rho(\boldsymbol{v})|,|w(\boldsymbol{v})|) .
$$

$$
\alpha^{*}(x)=|w|^{-1}|\rho|^{2-2 n} \rho^{*}\left(\eta^{z} \wedge v_{n-2}^{z}\right) \wedge(w d \bar{w}+\bar{w} d w) .
$$

Moreover, if $M$ is an (n-1)-dimensional smooth complex submanifold of $G$ and $\iota_{M}: M \rightarrow G$ is the inclusion, then

$$
\left|\iota_{M}^{*} \alpha^{*}(x)\right| \leqq \frac{1}{2}|\rho|^{3-2 n} \iota_{M}^{*}\left(v_{n-1}^{v}\right) .
$$

Finally, if $\nu$ is a nonnegative divisor on the open subset ${ }^{*} U$ of $G, M=\Re(\mathfrak{M}(\nu))$, and $\iota_{M}: M \rightarrow G$ is the inclusion, then

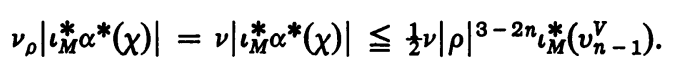

Proof. We have $\alpha^{*}(\chi)=\alpha^{*}\left(\pi_{1}^{*}(\sigma)\right) \wedge \alpha^{*}\left(\pi_{2}^{*}(d r)\right)$. Now $2|w| d|w|=d|w|^{2}=d(w \bar{w})$ $=w d \bar{w}+\bar{w} d w ;$ hence

$$
\alpha^{*}\left(\pi_{2}^{*}(d r)\right)=d\left(r \circ \pi_{2} \circ \alpha\right)=d|w|=\frac{1}{2}|w|^{-1}(w d \bar{w}+\bar{w} d w) .
$$


Let $\theta=\theta^{z}: Z-\{0\} \rightarrow S$ and $\psi=\psi^{z}: Z-\{0\} \rightarrow R$. By 3.1,

$$
\begin{aligned}
\alpha^{*}\left(\pi_{1}^{*}(\sigma)\right) & =\left(\pi_{1} \circ \alpha\right)^{*}(\sigma)=(\theta \circ \rho)^{*}(\sigma)=\rho^{*}\left(\theta^{*}(\sigma)\right) \\
& =\rho^{*}\left(\left(2 / \psi^{2 n-2}\right) \eta^{Z} \wedge v_{n-2}^{Z}\right) \\
& =2|\rho|^{2-2 n} \rho^{*}\left(\eta^{Z} \wedge v_{n-2}^{Z}\right) .
\end{aligned}
$$

Therefore

$$
\alpha^{*}(\chi)=|w|^{-1}|\rho|^{2-2 n} \rho^{*}\left(\eta^{Z} \wedge v_{n-2}^{Z}\right) \wedge(w d \bar{w}+\bar{w} d w)
$$

We have

$$
\eta^{z}=\frac{1}{4} i\left[\bar{\partial}\left(\psi^{2}\right)-\partial\left(\psi^{2}\right)\right]=-\frac{1}{2} i \psi(\partial \psi-\bar{\partial} \psi) .
$$

Define $\phi=\psi \circ \rho=|\rho|: V \rightarrow \boldsymbol{R}$. Then

$$
\alpha^{*}(\chi)=-\frac{1}{2} i|w|^{-1}|\rho|^{3-2 n}(\partial \phi-\bar{\partial} \phi) \wedge(w d \bar{w}+\bar{w} d w) \wedge \rho^{*}\left(v_{n-2}^{Z}\right) .
$$

It is well known that

$$
v_{n-1}^{V}=\rho^{*}\left(v_{n-1}^{Z}\right)+\frac{1}{2} i d w \wedge d \bar{w} \wedge \rho^{*}\left(v_{n-2}^{Z}\right) .
$$

Let $M$ be an (n-1)-dimensional smooth complex submanifold of $G$, and let $\iota_{M}: M \rightarrow G$ be the inclusion. Then

$$
\iota_{M}^{*} \alpha^{*}(\chi)=-\frac{1}{2} i|w|^{-1}|\rho|^{3-2 n_{\iota}^{*}}\left((w \partial \phi \wedge d \bar{w}-\bar{w} \bar{\partial} \phi \wedge d w) \wedge \rho^{*}\left(v_{n-2}^{Z}\right)\right) .
$$

Note that $\gamma=\iota_{M}^{*}(2 \partial \phi+(\bar{w} /|w|) d w)$ has bidegree $(1,0)$ and that $\iota_{M}^{*} \rho^{*}\left(v_{n-2}^{Z}\right)$ is semipositive definite. According to 6.1,

$$
\frac{1}{2} i \gamma \wedge \bar{\gamma} \wedge \iota_{M}^{*} \rho^{*}\left(v_{n-2}^{Z}\right) \geqq 0 .
$$

But

$\frac{1}{2} i \gamma \wedge \bar{\gamma} \wedge \iota_{M}^{*} \rho^{*}\left(v_{n-2}^{Z}\right)$

$$
\begin{aligned}
&= \frac{1}{2} i_{M}^{*}(4 \partial \phi \wedge \bar{\partial} \phi+d w \wedge d \bar{w}+2(w /|w|) \partial \phi \wedge d \bar{w}-2(\bar{w}|| w \mid) \bar{\partial} \phi \wedge d w) \\
& \wedge \iota_{M}^{*} \rho^{*}\left(v_{n-2}^{Z}\right) \\
&= \iota_{M}^{*} \rho^{*}\left(2 i \partial \psi \wedge \bar{\partial} \psi \wedge v_{n-2}^{Z}\right)+\iota_{M}^{*}\left(\frac{1}{2} i d w \wedge d \bar{w} \wedge \rho^{*}\left(v_{n-2}^{Z}\right)\right) \\
&+\iota_{M}^{*}\left(i|w|^{-1}(w \partial \phi \wedge d \bar{w}-\bar{w} \bar{\partial} \phi \wedge d w) \wedge \rho^{*}\left(v_{n-2}^{Z}\right)\right) \\
&= \iota \\
& \iota_{M}^{*} \rho^{*}\left(v_{n-1}^{Z}\right)+\iota_{M}^{*}\left(\frac{1}{2} i d w \wedge d \bar{w} \wedge \rho^{*}\left(v_{n-2}^{Z}\right)\right)-2|\rho|^{2 n-3} \iota_{M}^{*} \alpha^{*}(\chi) \\
&= \iota_{M}^{*}\left(v_{n-1}^{V}\right)-2|\rho|^{2 n-3} \iota_{M}^{*} \alpha^{*}(\chi) .
\end{aligned}
$$

Hence

$$
\iota_{M}^{*} \alpha^{*}(\alpha) \leqq \frac{1}{2}|\rho|^{3-2 n} \iota_{M}^{*}\left(v_{n-1}^{V}\right) .
$$

Letting $\delta=\iota_{M}^{*}(2 \partial \phi-(\bar{w} /|w|) d w)$ and proceeding as with $\gamma$, we obtain

$$
0 \leqq \frac{1}{2} i \delta \wedge \delta \wedge \iota_{M}^{*} \rho^{*}\left(v_{n-2}^{Z}\right)=\iota_{M}^{*}\left(v_{n-1}^{V}\right)+2|\rho|^{2 n-3} \iota_{M}^{*} \alpha^{*}(\chi) .
$$

Hence

$$
-\iota_{M}^{*} \alpha^{*}(\chi) \leqq \frac{1}{2}|\rho|^{3-2 n} \iota_{M}^{*}\left(v_{n-1}^{V}\right),
$$

which completes the proof of the inequality stated in this lemma. 
Let $\nu$ be a nonnegative divisor on the open subset $U$ of $G$, define $M=\Re(\mathfrak{M}(\nu))$, and let $\iota_{M}: M \rightarrow G$ be the inclusion. Take $v \in M$. If $d\left(\rho \circ \iota_{M}\right)$ has complex rank $n-1$ at $v$, then by $2.1, \nu(v)=\nu_{\rho}(v)$; hence $\nu_{\rho}\left|\iota_{M}^{*} \alpha^{*}(\chi)\right|=\nu\left|\iota_{M}^{*} \alpha^{*}(\chi)\right|$ at $v$. If the complex rank of $d\left(\rho \circ \iota_{M}\right)$ is less than $n-1$ at $v$, then the real rank is at most $2 n-4$ at $v$; but $\eta^{z} \wedge v_{n-2}^{z}$ has degree $2 n-3$; by $1.1, \iota_{M}^{*} \rho^{*}\left(\eta^{z} \wedge v_{n-2}^{z}\right)(v)=0$; hence $\iota_{M}^{*} \alpha^{*}(\chi)(v)=0$, and $\nu_{\rho}\left|\iota_{M}^{*} \alpha^{*}(\chi)\right|=0=\nu\left|\iota_{M}^{*} \alpha^{*}(\chi)\right|$ at $v$, Q.E.D.

Integrating, we obtain

LemMa 6.3. Let $c$ be a unit vector in the $n$-dimensional unitary space $V$. Define $Z=E(c)$, and let $\rho=\rho_{c}: V \rightarrow Z$. Define $S=S^{z}=\{z|z \in Z| z \mid,=1\}$, and let $\sigma=\sigma^{z}$ be the euclidean volume element of $S$. Let $0<R_{0}<R, 0<R^{\prime \prime}<R^{\prime}$, and define

$$
U=\left\{z+w c\left|z \in Z, R_{0}<\right| z\left|<R, R^{\prime \prime}<\right| w \mid<R^{\prime}\right\} .
$$

Let $\nu$ be a nonnegative divisor on $U$. Then

$$
\int_{R^{\prime \prime}}^{R^{\prime}}\left(\int_{a \in S} \sum_{R_{0}<t<R} \sum_{|w|=r} \nu_{\rho}(t a+w c) \sigma(a)\right) d r \leqq \frac{1}{2} R_{0}^{3-2 n} V_{v}(U) .
$$

Proof. Let $r: R \rightarrow R$ be the identity function. Let $\pi_{1}: S \times R \rightarrow S, \pi_{2}: S \times R \rightarrow R$ be the projections, and define

$$
\chi=\pi_{1}^{*}(\sigma) \wedge \pi_{2}^{*}(d r)
$$

Define $\alpha: U \rightarrow S \times R$ by

$$
\alpha(\boldsymbol{v})=(\rho(\boldsymbol{v}) /|\rho(\boldsymbol{v})|,|(\boldsymbol{v} \mid \boldsymbol{c})|) .
$$

Define $M=\mathfrak{R}(\mathfrak{M}(\nu))$, let $\iota_{M}: M \rightarrow U$ be the inclusion, and define $\beta=\alpha \mid M=\alpha \circ \iota_{M}$. According to 1.4 and 6.2 ,

$$
\begin{aligned}
\int_{y \in S \times R} \sum_{v \in \beta^{-1}(y)} \nu_{\rho}(v) \chi(y) & =\int_{M} \nu_{\rho}\left|\beta^{*}(\chi)\right| \\
& \leqq \frac{1}{2} \int_{M} \nu|\rho|^{3-2 n} \iota_{M}^{*}\left(v_{n-1}^{V}\right) \\
& \leqq \frac{1}{2} R_{0}^{3-2 n} V_{\nu}(U) .
\end{aligned}
$$

Using the fact that $\alpha(\mathfrak{M}(\nu)-M)$ has measure zero in $S \times R$, and then applying Fubini's theorem (1.2), we obtain

$$
\begin{aligned}
\int_{y \in S \times R} \sum_{v \in \beta^{-1}(y)} \nu_{\rho}(v) \cdot \chi(y) & =\int_{y \in S \times R} \sum_{v \in \alpha^{-1}(y)} \nu_{\rho}(v) \chi(y) \\
& =\int_{R^{\prime \prime}}^{R^{\prime}}\left(\int_{a \in S} \sum_{R_{0}<t<R} \sum_{|w|=r} \nu_{\rho}(t a+w c) \sigma(a)\right) d r \text {, Q.E.D. }
\end{aligned}
$$

7. Applying the estimates. We begin by giving coordinate-free definitions of the geometrical figures considered in the introduction. Let $c$ be a unit vector in the unitary space $V$. For $0<R_{0}<R, 0<R^{\prime \prime}<R^{\prime}$, we define

$$
\Omega\left(c ; R, R^{\prime}\right)=\left\{z+w c|z \in E(c),| z|<R,| w \mid<R^{\prime}\right\},
$$

$\Gamma\left(\boldsymbol{c} ; R_{0}, R ; R^{\prime \prime}, R^{\prime}\right)=\Omega\left(\boldsymbol{c} ; R_{0}, R^{\prime}\right) \cup\left\{z+w c|z \in E(\boldsymbol{c})| z,\left|<R, R^{\prime \prime}<\right| w \mid<R^{\prime}\right\}$. 
We now combine our estimates to prove

LeMma 7.1. Let $0<P_{0}<P_{1}<P, 0<P^{\prime \prime}<P_{1}^{\prime}<P^{\prime}$, and let $n$ be an integer greater than 1. Define

$$
\begin{aligned}
L & =L\left(n ; P_{0}, P_{1} ; P_{1}^{\prime}, P^{\prime}\right) \\
& =1+(2 n-2)^{-1}\left(2 P_{1} / P_{0}\right)^{2 n-2}\left(1+\frac{1}{4} P_{0}\left(P^{\prime}-P_{1}^{\prime}\right)^{-1}\right) .
\end{aligned}
$$

Let $c$ be a unit vector in the n-dimensional unitary space $V$. Define $Z=E(c)$; and let $\rho=\rho_{c}: V \rightarrow Z$. Let $\nu$ be a nonnegative divisor on $\Omega\left(c ; P, P^{\prime}\right)$, and define

$$
M=\mathfrak{R}(\mathfrak{M}(\nu)) \cap \Omega\left(c ; P_{1}, P_{1}^{\prime}\right) .
$$

Then

$$
\int_{M} \nu \rho^{*}\left(v_{n-1}^{Z}\right) \leqq L \cdot V_{v}\left(\Gamma\left(c ; P_{0}, P ; P^{\prime \prime}, P^{\prime}\right)\right) .
$$

Proof. Define $V_{0}=V_{v}\left(\Gamma\left(c ; P_{0}, P ; P^{\prime \prime}, P^{\prime}\right)\right)$. Define $S=S^{z}=\{z|z \in Z| z \mid,=1\}$, and let $\sigma=\sigma^{z}$ be the euclidean volume element of $S$. Define

$$
\begin{aligned}
U & =\left\{z+w c\left|z \in Z, \frac{1}{2} P_{0}<\right| z\left|<P_{1}, P_{1}^{\prime}<\right| w \mid<P^{\prime}\right\}, \\
a(r) & =\int_{a \in S} \sum_{t P_{0}} \sum_{t<P_{1}} v_{|w|=r}(t a+w c) \sigma(a) .
\end{aligned}
$$

Using 6.3 (the third estimate) and the fact that $U \subset \Gamma\left(c ; P_{0}, P ; P^{\prime \prime}, P^{\prime}\right)$, we obtain

$$
\int_{P_{1}^{\prime}}^{P^{\prime}} a(r) d r \leqq \frac{1}{2}\left(\frac{1}{2} P_{0}\right)^{3-2 n} V_{v}(U) \leqq \frac{1}{2}\left(2 / P_{0}\right)^{2 n-3} V_{0}
$$

Therefore there exists $R^{\prime}$ with $P_{1}^{\prime}<R^{\prime}<P^{\prime}$ and

$$
a\left(R^{\prime}\right) \leqq\left(P^{\prime}-P_{1}^{\prime}\right)^{-1} \frac{1}{2}\left(2 / P_{0}\right)^{2 n-3} V_{0} .
$$

Define

$$
\begin{aligned}
A & =\left\{z\left|z \in Z, \frac{1}{2} P_{0}<\right| z \mid<P_{0}\right\}, \\
b(r) & =\int_{a \in S} \sum_{|w|<P^{\prime}} \nu_{\rho}(r a+w c) \sigma(a) .
\end{aligned}
$$

Using 3.2, 4.1 (the first estimate), and the fact that

$$
\left\{z+w c|z \in A,| w \mid<P^{\prime}\right\} \subset \Gamma\left(c ; P_{0}, P ; P^{\prime \prime}, P^{\prime}\right),
$$

we obtain

$$
\int_{\ddagger P_{0}}^{P_{0}} b(r) r^{2 n-3} d r=\int_{z \in A} \sum_{|w|<P^{\prime}} \nu_{\rho}(z+w c) v_{n-1}^{z}(z) \leqq V_{0} .
$$

Therefore there exists $R_{0}$ with $\frac{1}{2} P_{0}<R_{0}<P_{0}$ and

$$
b\left(R_{0}\right) R_{0}^{2 n-3} \leqq\left(P_{0}-\frac{1}{2} P_{0}\right)^{-1} V_{0} .
$$

It follows that

$$
b\left(R_{0}\right) \leqq\left(2 / P_{0}\right)^{2 n-2} V_{0}
$$


Define $H=\left\{z\left|z \in Z, R_{0}<\right| z \mid<P_{1}\right\}$. We have $M \subset M^{\prime} \cup M^{\prime \prime}$ where

$$
\begin{aligned}
& M^{\prime}=\mathfrak{R}(\mathfrak{M}(\nu)) \cap \Gamma\left(c ; P_{0}, P ; P^{\prime \prime}, P^{\prime}\right), \\
& M^{\prime \prime}=\mathfrak{R}(\mathfrak{M}(\nu)) \cap\left\{z+w c|z \in H,| w \mid<R^{\prime}\right\} .
\end{aligned}
$$

Hence

$$
\int_{M} \nu \rho^{*}\left(v_{n-1}^{Z}\right) \leqq \int_{M^{\prime}} \nu \rho^{*}\left(v_{n-1}^{Z}\right)+\int_{M^{\nu}} \nu \rho^{*}\left(v_{n-1}^{Z}\right)
$$

Clearly

$$
\int_{M^{\prime}} \nu \rho^{*}\left(v_{n-1}^{Z}\right) \leqq V_{0}
$$

Applying 4.1 (the first estimate), 5.2 (the second estimate), and 3.2, we see that

$$
\begin{aligned}
\int_{M^{\prime \prime}} \nu \rho^{*}\left(v_{n-1}^{z}\right)= & \int_{z \in H} \sum_{|w|<R^{\prime}} \nu_{\rho}(z+w c) v_{n-1}^{z}(z) \\
\leqq & \int_{z \in H} \sum_{|w|<R^{\prime}} v_{\rho}\left(R_{0} z|| z \mid+w c\right) v_{n-1}^{z}(z) \\
& +\int_{z \in H} \sum_{R_{0} \leqq t \leqq|z|} \sum_{|w|=R^{\prime}} v_{\rho}(t z|| z \mid+w c) v_{n-1}^{z}(z) \\
\leqq & \int_{z \in H} \sum_{|w|<R^{\prime}} v_{\rho}\left(R_{0} z /|z|+w c\right) v_{n-1}^{z}(z) \\
& +\int_{z \in H} \sum_{R_{0} \leqq t<P_{1}} \sum_{|w|=R^{\prime}} \nu_{\rho}(t z /|z|+w c) v_{n-1}^{z}(z) \\
= & (2 n-2)^{-1}\left(P_{1}^{2 n-2}-R_{0}^{2 n-2}\right) \\
& \times\left[\int_{a \in S} \sum_{|w|<R^{\prime}} \nu_{\rho}\left(R_{0} a+w c\right) \sigma(a)+\int_{a \in S R_{0} \leqq t<P_{1}} \sum_{|w|=R^{\prime}} \nu_{\rho}(t a+w c) \sigma(a)\right] \\
\leqq & (2 n-2)^{-1} P_{1}^{2 n-2}\left[b\left(R_{0}\right)+a\left(R^{\prime}\right)\right] \\
\leqq & (2 n-2)^{-1} P_{1}^{2 n-2}\left[\left(2 / P_{0}\right)^{2 n-2} V_{0}+\left(P^{\prime}-P_{1}^{\prime}\right)^{-1} \frac{1}{2}\left(2 / P_{0}\right)^{2 n-3} V_{0}\right] \\
= & (L-1) V_{0} .
\end{aligned}
$$

Therefore

$$
\int_{M} \nu \rho^{*}\left(v_{n-1}^{z}\right) \leqq L \cdot V_{0}
$$

8. Wiggling. In the last section we established an estimate for one of the $n$ "components" of the volume of a divisor. Since estimation of the other $n-1$ "components" appears difficult, we avoid it by means of the "wiggling" lemmas. In proving our first wiggling lemma we use the existence of "normal" vectors:

LEMMA 8.1. Let $V$ be an n-dimensional unitary space. For each unit vector $c \in V$, define $z_{c}: V \rightarrow C$ by $z_{c}(v)=(v \mid c)$. Let $M$ be an $(n-1)$-dimensional smooth complex 
submanifold of $V$, and let $\iota_{M}: M \rightarrow V$ be the inclusion. Take $b \in M$. Then there exists a unit vector $\boldsymbol{n} \in V$ with $\iota_{M}^{*}\left(d z_{n}\right)(\boldsymbol{b})=0$.

Proof. Let $c_{1}, \ldots, c_{n}$ be a basis for $V$. Then $\iota_{M}^{*}\left(d z_{c_{1}}\right)(\boldsymbol{b}), \ldots, \iota_{M}^{*}\left(d z_{c_{n}}\right)(\boldsymbol{b})$ are linearly dependent, i.e., there exists $\left(a_{1}, \ldots, a_{n}\right) \in C^{n}-\{0\}$ such that

$$
\sum a_{j} \iota_{M}^{*}\left(d z_{c_{j}}\right)(b)=0 .
$$

Define $\boldsymbol{n}=\sum \bar{a}_{j} \boldsymbol{c}_{j} /\left|\sum \bar{a}_{j} \boldsymbol{c}_{j}\right|$. Then $|\boldsymbol{n}|=1$, and a straightforward computation shows that $\iota_{M}^{*}\left(d z_{n}\right)(\boldsymbol{b})=0$, Q.E.D.

The first wiggling lemma states that estimates can be obtained without assuming that the basis is orthonormal. More precisely,

LEMMA 8.2. Let $c_{1}, \ldots, c_{n}$ be a basis for the unitary space $V$. Suppose that each $c_{j}$ is a unit vector, let $Z_{j}=E\left(c_{j}\right)$, and define $\rho_{j}=\rho_{c_{j}}: V \rightarrow Z_{j}$. Define

$$
K=\operatorname{Min}\left\{\sum_{j}\left|\left(\boldsymbol{e} \mid \boldsymbol{c}_{j}\right)\right|^{2}|\boldsymbol{e} \in V,| \boldsymbol{e} \mid=1\right\}>0 .
$$

Let $M$ be an (n-1)-dimensional smooth complex submanifold of $V$, and let $\iota_{M}: M \rightarrow V$ be the inclusion. Then

$$
\iota_{M}^{*}\left(v_{n-1}^{v}\right) \leqq K^{-1} \iota_{M}^{*}\left(\sum_{j} \rho_{j}^{*}\left(v_{n-1}^{Z_{j}}\right)\right) .
$$

Proof. Take $\boldsymbol{b} \in M$. Let $\boldsymbol{n}$ be the unit vector given by 8.1. Define $z: V \rightarrow C$ by $z(v)=(v \mid n)$; then $\iota_{M}^{*}(d z)(b)=0$. Let $e_{1}, \ldots, e_{n}$ be an orthonormal basis for $V$ with $\boldsymbol{e}_{n}=n$. Define $z_{j}: V \rightarrow C$ by $z_{j}(v)=\left(v \mid e_{j}\right), j=1, \ldots, n$.

Let $c$ be a unit vector in $V$. Define $Z=E(c)$, and let $\rho=\rho_{c}: V \rightarrow Z$. Define $w: V \rightarrow C$ by $w(v)=(v \mid c)$. In coordinate notation it is clear that

We have

$$
v_{n-1}^{V}=\rho^{*}\left(v_{n-1}^{Z}\right)+\frac{1}{2} i d w \wedge d \bar{w} \wedge v_{n-2}^{V} \text {. }
$$

Since $\iota_{M}^{*}\left(d z_{n}\right)(\boldsymbol{b})=0$,

$$
\begin{aligned}
w(\boldsymbol{v}) & =\left(\sum z_{j}(\boldsymbol{v}) \boldsymbol{e}_{j} \mid \boldsymbol{c}\right)=\sum\left(\boldsymbol{e}_{j} \mid \boldsymbol{c}\right) z_{j}(\boldsymbol{v}) \\
d w & =\sum\left(\boldsymbol{e}_{j} \mid \boldsymbol{c}\right) d z_{j}, \quad d \bar{w}=\sum\left(\boldsymbol{c} \mid \boldsymbol{e}_{j}\right) d \bar{z}_{j} .
\end{aligned}
$$

$\iota_{M}^{*}\left(\frac{1}{2} i d w \wedge d \bar{w} \wedge v_{n-2}^{V}\right)(b)$

$$
\begin{aligned}
= & \iota_{M}^{*}\left[\frac{1}{2} i\left(\sum_{j=1}^{n-1}\left(\boldsymbol{e}_{j} \mid \boldsymbol{c}\right) d z_{j}\right) \wedge\left(\sum_{j=1}^{n-1}\left(\boldsymbol{c} \mid \boldsymbol{e}_{j}\right) d \bar{z}_{j}\right)\right. \\
& \left.\left.\wedge\left(\sum_{j=1}^{n-1}\left(\frac{1}{2} i\right)^{n-2} d z_{1} \wedge d \bar{z}_{1} \wedge \cdots \|^{j} \cdots \wedge d z_{n-1} \wedge d \bar{z}_{n-1}\right)\right)\right](\boldsymbol{b}) \\
= & \iota_{M}^{*}\left(\sum_{j=1}^{n-1}\left(\frac{1}{2} i\right)^{n-1}\left|\left(\boldsymbol{e}_{j} \mid \boldsymbol{c}\right)\right|^{2} d z_{1} \wedge d \bar{z}_{1} \wedge \cdots \wedge d z_{n-1} \wedge d \bar{z}_{n-1}\right)(\boldsymbol{b}) \\
= & \left(\sum_{j=1}^{n-1}\left|\left(\boldsymbol{e}_{j} \mid \boldsymbol{c}\right)\right|^{2}\right) \iota_{M}^{*}\left(v_{n-1}^{V}\right)(\boldsymbol{b}) .
\end{aligned}
$$


Therefore

$$
\begin{aligned}
\iota_{M}^{*} \rho^{*}\left(v_{n-1}^{Z}\right)(\boldsymbol{b}) & =\iota_{M}^{*}\left(v_{n-1}^{V}-\frac{1}{2} i d w \wedge d \bar{w} \wedge v_{n-2}^{V}\right)(\boldsymbol{b}) \\
& =\left(1-\sum_{j=1}^{n-1}\left|\left(\boldsymbol{e}_{j} \mid \boldsymbol{c}\right)\right|^{2}\right) \iota_{M}^{*}\left(v_{n-1}^{V}\right)(\boldsymbol{b}) \\
& =\left|\left(\boldsymbol{e}_{n} \mid \boldsymbol{c}\right)\right|^{2} \iota_{M}^{*}\left(v_{n-1}^{V}\right)(\boldsymbol{b}) .
\end{aligned}
$$

Here we have used the fact that $|c|=1$. Since $c$ was an arbitrary unit vector in $V$, we have

$$
\begin{aligned}
\iota_{M}^{*}\left(\sum_{j} \rho_{j}\left(v_{n-1}^{Z_{j}}\right)\right)(\boldsymbol{b}) & =\left(\sum_{j}\left|\left(\boldsymbol{e}_{n} \mid \boldsymbol{c}_{j}\right)\right|^{2}\right) \iota_{M}^{*}\left(v_{n-1}^{V}\right)(\boldsymbol{b}) \\
& \geqq K \iota_{M}^{*}\left(v_{n-1}^{V}\right)(\boldsymbol{b}),
\end{aligned}
$$

The second wiggling lemma shows that a small change in the unit vector $c$ does not seriously disturb the geometrical figures of $\$ 7$.

LEMMA 8.3. Let $c$ be a unit vector in the unitary space $V$. Let $0<R_{0}<R_{1}<R$, $0<R^{\prime \prime}<R_{1}^{\prime}<R^{\prime}$. Then there exist constants $\delta>0,0<P_{0}<P_{1}<P, 0<P^{\prime \prime}<P_{1}^{\prime}<P^{\prime}$, such that

$$
\begin{aligned}
\Gamma\left(b ; P_{0}, P ; P^{\prime \prime}, P^{\prime}\right) & \subset \Gamma\left(c ; R_{0}, R ; R^{\prime \prime}, R^{\prime}\right), \\
\Omega\left(b ; P, P^{\prime}\right) & \subset \Omega\left(c ; R, R^{\prime}\right), \\
\Omega\left(c ; R_{1}, R_{1}^{\prime}\right) & \subset \Omega\left(b ; P_{1}, P_{1}^{\prime}\right),
\end{aligned}
$$

whenever $\boldsymbol{b}$ is a unit vector in $V$ with $|\boldsymbol{b}-\boldsymbol{c}|<\delta$.

Proof. Define $\varepsilon=\operatorname{Min}\left(R-R_{1}, R^{\prime}-R_{1}^{\prime}, R_{0}\right)$. Define $\delta=\varepsilon / 6\left(R+R^{\prime}\right)$,

$$
\begin{array}{llrl}
P_{0}=R_{0}-\frac{1}{3} \varepsilon, & P_{1}=R_{1}+\frac{1}{3} \varepsilon, & P & =R-\frac{1}{3} \varepsilon, \\
P^{\prime \prime}=R^{\prime \prime}+\frac{1}{3} \varepsilon, & P_{1}^{\prime}=R_{1}^{\prime}+\frac{1}{3} \varepsilon, & P^{\prime}=R^{\prime}-\frac{1}{3} \varepsilon .
\end{array}
$$

Applying the triangle inequality many times will complete the proof; we omit this tedious exercise.

9. The lemma. We are finally prepared to prove the main result of this paper, a generalization to $n$ dimensions of a result of K. Oka [8, p. 11].

LEMMA 9.1. Let $c$ be a unit vector in the n-dimensional unitary space $V$. Let $0<R_{0}<R_{1}<R, 0<R^{\prime \prime}<R_{1}^{\prime}<R^{\prime}$. Then there exists a constant

$$
C=C\left(n ; R_{0}, R_{1}, R ; R^{\prime \prime}, R_{1}^{\prime}, R^{\prime}\right)
$$

such that

$$
V_{\nu}\left(\Omega\left(c ; R_{1}, R_{1}^{\prime}\right)\right) \leqq C \cdot V_{\nu}\left(\Gamma\left(c ; R_{0}, R ; R^{\prime \prime}, R^{\prime}\right)\right)
$$

for every nonnegative divisor $v$ on $\Omega\left(c ; R, R^{\prime}\right)$. 
Proof. Let $\delta>0,0<P_{0}<P_{1}<P, 0<P^{\prime \prime}<P_{1}^{\prime}<P^{\prime}$ be the constants given by 8.3. For each unit vector $\boldsymbol{b} \in V$ with $|\boldsymbol{b}-\boldsymbol{c}|<\delta$, we have

$$
\begin{aligned}
\Gamma\left(b ; P_{0}, P ; P^{\prime \prime}, P^{\prime}\right) & \subset \Gamma\left(c ; R_{0}, R ; R^{\prime \prime}, R^{\prime}\right), \\
\Omega\left(b ; P, P^{\prime}\right) & \subset \Omega\left(c ; R, R^{\prime}\right), \\
\Omega\left(c ; R_{1}, R_{1}^{\prime}\right) & \subset \Omega\left(b, P_{1}, P_{1}^{\prime}\right) .
\end{aligned}
$$

Let $c_{1}, \ldots, c_{n}$ be a basis for $V$ with $\left|c_{j}\right|=1$ and $\left|c_{j}-c\right|<\delta, j=1, \ldots, n$. Let

$$
K=\operatorname{Min}\left\{\sum_{j}\left|\left(e \mid c_{j}\right)\right|^{2}|e \in V,| e \mid=1\right\}>0 .
$$

Let $Z_{j}=E\left(c_{j}\right)$ and define $\rho_{j}=\rho_{c_{j}}: V \rightarrow Z_{j}$. According to 8.2,

$$
\iota_{M}^{*}\left(v_{n-1}^{v}\right) \leqq K^{-1} \iota_{M}^{*}\left(\sum_{j} \rho_{j}^{*}\left(v_{n-1}^{Z_{j}}\right)\right)
$$

whenever $M$ is an $(n-1)$-dimensional smooth complex submanifold of $V$ and $\iota_{M}: M \rightarrow V$ is the inclusion. Let $L=L\left(n ; P_{0}, P_{1} ; P_{1}^{\prime}, P^{\prime}\right)$ be the constant given in 7.1. Define

$$
C=C\left(n ; R_{0}, R_{1}, R ; R^{\prime \prime}, R_{1}^{\prime}, R^{\prime}\right)=n L / K .
$$

Let $\nu$ be a nonnegative divisor on $\Omega\left(c ; R, R^{\prime}\right)$. Define

$$
\begin{aligned}
M & =\mathfrak{R}(\mathfrak{M}(\nu)) \cap \Omega\left(c ; R_{1}, R_{1}^{\prime}\right), \\
M_{j} & =\Re(\mathfrak{M}(\nu)) \cap \Omega\left(c_{j} ; P_{1}, P_{1}^{\prime}\right), \quad j=1, \ldots, n .
\end{aligned}
$$

Then, using 7.1 we obtain

$$
\begin{aligned}
V_{v}\left(\Omega\left(c ; R_{1}, R_{1}^{\prime}\right)\right) & =\int_{M} \nu v_{n-1}^{v} \\
& \leqq K^{-1} \sum_{j} \int_{M} \nu \rho_{j}^{*}\left(v_{n-1}^{Z_{j}}\right) \\
& \leqq K^{-1} \sum_{j} \int_{M,} \nu \rho_{j}^{*}\left(v_{n-1}^{z_{j}}\right) \\
& \leqq K^{-1} \sum_{j} L \cdot V_{v}\left(\Gamma\left(c_{j} ; P_{0}, P ; P^{\prime \prime}, P^{\prime}\right)\right) \\
& \leqq(n L / K) V_{v}\left(\Gamma\left(c ; R_{0}, R ; R^{\prime \prime}, R^{\prime}\right)\right),
\end{aligned}
$$

10. Stein manifolds. We assume knowledge of the basic facts about Stein manifolds listed by Gunning and Rossi [5, Theorem 4, p. 283]. In this section we supply an answer to the question: When is an open subset of a Stein manifold again a Stein manifold? We will use the answer given by H. J. Bremermann [1]:

Proposition 10.1. Let $D$ be an open subset of the Stein manifold G. Then $D$ is a Stein manifold if and only if it is locally a Stein manifold, i.e., each point of $\bar{D}$ has an open neighborhood $U$ such that $U \cap D$ is a Stein manifold. 
Let $G$ and $H$ be complex manifolds, and let $A \subset G$. We say that $\tau: A \rightarrow H$ is holomorphic (resp. biholomorphic) iff there exists an open neighborhood $U$ of $A$ and a holomorphic (resp. biholomorphic) extension $\tau_{1}: U \rightarrow H$ (resp. $\tau_{1}: U$ $\left.\rightarrow \tau_{1}(U) \subset H\right)$ of $\tau$.

In $C^{n}$ we define the compact sets

$$
\begin{aligned}
& \Omega=\left\{\left.\left(z_{1}, \ldots, z_{n}\right)|| z_{1}\right|^{2}+\cdots+\left|z_{n-1}\right|^{2} \leqq 1,\left|z_{n}\right| \leqq 1\right\}, \\
& \Gamma=\left\{\left.\left(z_{1}, \ldots, z_{n}\right)|| z_{1}\right|^{2}+\cdots+\left|z_{n-1}\right|^{2} \leqq 1,\left|z_{n}\right|=1\right\} \cup\left\{\left(0, \ldots, 0, z_{n}\right)|| z_{n} \mid \leqq 1\right\} .
\end{aligned}
$$

The Cauchy integral formula allows us to extend each holomorphic function $f$ on $\Gamma$ to a holomorphic function $f_{1}$ on $\Omega$ given by

$$
f_{1}\left(z_{1}, \ldots, z_{n}\right)=\frac{1}{2 \pi i} \int_{|\zeta|=1} f\left(z_{1}, \ldots, z_{n-1}, \zeta\right) \frac{d \zeta}{\zeta-z_{n}}
$$

for $\left|z_{n}\right|<1$.

Our answer to the question of this section appears below. It seems to be wellknown but unpublished. The author is indebted to Professor Hans Grauert for the method used in part (b) (the difficult part).

Proposition 10.2. Let $D$ be an open subset of the n-dimensional Stein manifold $G$. Then $D$ is a Stein manifold if and only if $\tau(\Omega) \subset D$ whenever $\tau: \Omega \rightarrow G$ is a biholomorphic map with $\tau(\Gamma) \subset D$.

Proof. (a) Suppose that $D$ is a Stein manifold. Let $\tau: \Omega \rightarrow G$ be a biholomorphic map with $K=\tau(\Gamma) \subset D$. If $f$ is a continuous function on the compact set $K$, we define

$$
\|f\|_{K}=\operatorname{Max}\{|f(x)| \mid x \in K\} .
$$

Let $\mathfrak{D}_{D}$ be the set of holomorphic functions defined on $D$. Since each holomorphic function on $\Gamma$ extends to a holomorphic function on $\Omega$, the same is true for $\tau(\Gamma)=K$ and $\tau(\Omega)$. By the maximum modulus theorem

$$
\hat{K}=\left\{x|x \in D,| f(x) \mid \leqq\|f\|_{K} \text { for all } f \in \mathfrak{D}_{D}\right\} \supset D \cap \tau(\Omega) \supset K \neq \varnothing .
$$

Since $D$ is a Stein manifold, $\hat{K}$ is compact. Hence

$$
D \cap \tau(\Omega)=\hat{K} \cap D \cap \tau(\Omega)=\hat{K} \cap \tau(\Omega)
$$

is open and closed in the connected set $\tau(\Omega)$. Therefore $\tau(\Omega)=D \cap \tau(\Omega)$, i.e., $\tau(\Omega) \subset D$.

(b) Suppose that $\tau(\Omega) \subset D$ whenever $\tau: \Omega \rightarrow G$ is a biholomorphic map with $\tau(\Gamma) \subset D$. We must prove that $D$ is a Stein manifold. In view of 10.1 , we may assume that $G$ is an open subset of $C^{n}$. Suppose that $D$ is not a Stein manifold. Then $D \neq C^{n}$. Let | $\mid$ be the euclidean norm on $C^{n}:|z|^{2}=\sum\left|z_{j}\right|^{2}$ if $z=\left(z_{1}, \ldots, z_{n}\right)$. Define $d: D \rightarrow R$ by

$$
d(\boldsymbol{z})=\operatorname{Min}\{|\boldsymbol{z}-\boldsymbol{w}| \mid \boldsymbol{w} \notin D\}
$$


Then $-\log d$ is not plurisubharmonic. Since $d$ is positive and continuous, $-\log d$ is continuous. Hence there exists a plane

$$
T=\left\{z \mid z=z_{0}+t e, t \in C\right\}
$$

where $z_{0} \in C^{n}, e \in C^{n},|e|=1$, such that

$$
(-\log d) \mid T \cap D
$$

is not subharmonic. By changing $z_{0}$ if necessary, there exists $r>0$ and a continuous function $h$ on

such that

$$
\bar{\Delta}=\left\{z\left|z=z_{0}+t e,\right| t \mid \leqq r\right\} \subset D
$$

(1) $h$ is harmonic on $\Delta=\left\{z\left|z=z_{0}+t e,\right| t \mid<r\right\}$,

(2) $-\log d(z) \leqq h(z)$ for $z \in \bar{\Delta}-\Delta$,

(3) $-\log d(a)>h(a)$ for some $a \in \Delta$.

By adding a small positive constant to $h$ and then taking $r$ slightly smaller, we may even assume that

$\left(1^{\prime}\right) h$ is defined and harmonic in an open neighborhood of $\bar{\Delta}$ in $T$.

Finally, we can change our coordinate system in $C^{n}$ so as to make $z_{0}=0$ and $r=1$. Let $g$ be a holomorphic function on $\bar{\Delta}$ with $-h=\Re e g$ (the real part of $g$ ). Define $f=e^{g}$. Then $f$ is nonzero and holomorphic on $\Delta$, and

$$
|f|=e^{\Re \mathrm{e} g}=e^{-h} \text {. }
$$

We can rewrite (2), (3) as

(2') $|f(z)|=e^{-h(z)} \leqq d(z)$ for $z \in \bar{\Delta}-\Delta$,

(3') $|f(a)|=e^{-h(a)}>d(a)$.

Now $\boldsymbol{a}=a \boldsymbol{e}$, where $|a|<1$. Take $\boldsymbol{b} \in \bar{D}-D$ with $d(\boldsymbol{a})=|\boldsymbol{a}-\boldsymbol{b}|$.

(i) If $b \in T$, then $|\boldsymbol{b}|>1$, i.e., $\boldsymbol{b}=b e$ where $|b|>1$. For $|t| \leqq 1$, define

$$
F(t)=f(t e) /(t-b) \text {. }
$$

Then $F$ is holomorphic, and for $|t|=1$,

$$
\begin{aligned}
|F(t)| & =|f(t e)| /|t-b| \leqq d(t e) /|t-b| \\
& \leqq|t e-b| /|t-b|=1 .
\end{aligned}
$$

By the maximum modulus principle, $|F(a)| \leqq 1$. But

$$
\begin{aligned}
|F(a)| & =|f(\boldsymbol{a})| /|a-b|>d(\boldsymbol{a}) /|a-b| \\
& =|\boldsymbol{a}-\boldsymbol{b}| /|a-b|=1,
\end{aligned}
$$

a contradiction.

(ii) If $\boldsymbol{b} \notin T$, then $\boldsymbol{e}$ and $\boldsymbol{c}=\boldsymbol{a}-\boldsymbol{b}$ are linearly independent. Define $\boldsymbol{c}_{1}=\boldsymbol{c} /|\boldsymbol{c}|$; take $c_{2}, \ldots, c_{n-1} \in C^{n}$ such that $c_{1}, \ldots, c_{n-1}$ are orthonormal and $c_{1}, \ldots, c_{n-1}, e$ are linearly independent. Define $\lambda=d(\boldsymbol{a}) / f(\boldsymbol{a})$. Then $0<|\lambda|<1$. Define $\tau: \Omega \rightarrow \boldsymbol{C}^{n}$ by

$$
\tau\left(z_{1}, \ldots, z_{n}\right)=z_{n} e-\lambda f\left(z_{n} e\right) \sum_{j=1}^{n-1} z_{j} c_{j} \text {. }
$$


In the coordinates given by $c_{1}, \ldots, c_{n-1}, e$, the Jacobian determinant of $\tau$ is $\left(-\lambda f\left(z_{n} e\right)\right)^{n-1} \neq 0$; moreover $\tau$ is one-to-one. Therefore $\tau: \Omega \rightarrow C^{n}$ is biholomorphic. Now

$$
\tau\left(0, \ldots, 0, z_{n}\right)=z_{n} \boldsymbol{e} \in \bar{\Delta} \subset D \quad \text { for }\left|z_{n}\right| \leqq 1 .
$$

Also, if $\left|z_{1}\right|^{2}+\cdots+\left|z_{n-1}\right|^{2} \leqq 1$ and $\left|z_{n}\right|=1$, then

$$
\begin{aligned}
\left|z_{n} e-\tau\left(z_{1}, \ldots, z_{n}\right)\right| & =\left|\lambda f\left(z_{n} e\right) \sum_{j=1}^{n-1} z_{j} \boldsymbol{c}_{j}\right| \\
& \leqq|\lambda|\left|f\left(z_{n} e\right)\right|<d\left(z_{n} e\right) ;
\end{aligned}
$$

hence $\tau\left(z_{1}, \ldots, z_{n}\right) \in D$. Therefore $\tau(\Gamma) \subset D \subset G$. Since $G \subset C^{n}$ is a Stein manifold, part (a) assures us that $\tau(\Omega) \subset G$. According to our basic assumption, $\tau(\Omega) \subset D$. In particular,

$$
\begin{aligned}
\tau(1,0, \ldots, 0, a) & =\boldsymbol{a}-\lambda f(\boldsymbol{a}) \boldsymbol{c}_{1} \\
& =\boldsymbol{a}-d(\boldsymbol{a}) \boldsymbol{c}_{1} \\
& =\boldsymbol{a}-|\boldsymbol{a}-\boldsymbol{b}| \cdot(\boldsymbol{a}-\boldsymbol{b}) /|\boldsymbol{a}-\boldsymbol{b}| \\
& =\boldsymbol{b}
\end{aligned}
$$

belongs to $D$, a contradiction, Q.E.D.

11. The set of points of the first kind. Oka [8, p. 11] introduced the concept of a point "de première espèce" given in

DefinITION 11.1. Let $\mathfrak{R}=\left\{\nu_{\lambda}\right\}_{\lambda \in \Lambda}$ be a family of nonnegative divisors on the $n$-dimensional complex manifold $G$, and let $A=\left\{a_{\lambda}\right\}_{\lambda \in \Lambda}$ be a family of nonnegative real numbers indexed by the same set $\Lambda$. A point $a \in G$ is said to be of the first kind (with respect to $\Re$ and $A$ ) iff there exists an open set $U$ in $C^{n}$ and a biholomorphic map $\alpha: U \rightarrow G$ such that

(1) $a \in \alpha(U)$,

(2) $\sup \left\{a_{\lambda} V_{v_{\lambda} \circ \alpha}(U) \mid \lambda \in \Lambda\right\}<\infty$.

(Since $\alpha$ is biholomorphic, $\alpha^{*}\left(\nu_{\lambda}\right)=\nu_{\lambda} \circ \alpha$.)

Let $\mathfrak{R}$ be a family of nonnegative divisors on the $n$-dimensional complex manifold $G$, and let $A$ be a family of nonnegative real numbers. The set $D$ of points of the first kind (w.r.t. $\mathfrak{R}$ and $A$ ) is clearly open. If $U$ is an open set in $C^{n}$ and $\alpha: U \rightarrow G$ is a biholomorphic map with $\alpha(U) \subset \subset D$, then

$$
\sup \left\{a_{\lambda} V_{v_{\lambda} \circ \alpha}(U) \mid \lambda \in \Lambda\right\}<\infty .
$$

We generalize a result of Oka $[8, \mathrm{pp} .11-12]$ in

THEOREM 11.2. Let $\mathfrak{R}=\left\{\nu_{\lambda}\right\}_{\lambda \in \Lambda}$ be a family of nonnegative divisors on the Stein manifold $G$, and let $A=\left\{a_{\lambda}\right\}_{\lambda \in \Lambda}$ be a family of nonnegative real numbers. Then

$$
D=\{z \mid z \in G, z \text { is of the first kind w.r.t. } \Re \text { and } A\}
$$

is a Stein manifold. 
Proof. Our criterion is 10.2. Let $n$ be the dimension of $G$, and let $\tau: \Omega \rightarrow G$ be a biholomorphic map with $\tau(\Gamma) \subset D$. Let $U$ be an open neighborhood of $\Omega$ in $C^{n}$ and $\tau_{1}: U \rightarrow \tau_{1}(U) \subset G$ be a biholomorphic extension of $\tau$. Define $c=(0, \ldots, 0,1)$ $\in C^{n}$. Take $0<R_{0}<1<R_{1}<R, 0<R^{\prime \prime}<1<R_{1}^{\prime}<R^{\prime}$ such that

$$
\begin{aligned}
& \Omega \subset \Omega\left(c ; R_{1}, R_{1}^{\prime}\right) \subset \Omega\left(c ; R, R^{\prime}\right) \subset U, \\
& \Gamma \subset \Gamma\left(c ; R_{0}, R ; R^{\prime \prime}, R^{\prime}\right) \subset \subset \tau_{1}^{-1}\left(D \cap \tau_{1}(U)\right) .
\end{aligned}
$$

Define

$$
L=\sup \left\{a_{\lambda} V_{v_{\lambda^{\circ} \tau_{1}}}\left(\Gamma\left(c ; R_{0}, R ; R^{\prime \prime}, R^{\prime}\right)\right) \mid \lambda \in \Lambda\right\}<\infty .
$$

Let $C=C\left(n ; R_{0}, R_{1}, R ; R^{\prime \prime}, R_{1}^{\prime}, R^{\prime}\right)$ be the constant given by 9.1 . Then

$$
a_{\lambda} V_{v_{\lambda} \circ \tau_{1}}\left(\Omega\left(c ; R_{1}, R_{1}^{\prime}\right)\right) \leqq C \cdot a_{\lambda} V_{v_{\lambda^{\circ}} \tau_{1}}\left(\Gamma\left(c ; R_{0}, R ; R^{\prime \prime}, R^{\prime}\right)\right) \leqq C \cdot L<\infty
$$

for $\lambda \in \Lambda$. Hence $\tau(\Omega) \subset D$, Q.E.D.

12. The normality domain. Stoll [13, Definition 2.6, p. 176] introduced a concept of convergence of divisors clearly equivalent to

Definition 12.1. A net $\left\{v_{j}\right\}_{j \in J}$ of nonnegative divisors on the complex manifold $G$ is said to be convergent iff each $a \in G$ possesses an open neighborhood $U$ on which there is a net $\left\{f_{j}\right\}_{j \in J}$ of holomorphic functions satisfying

(1) $\nu_{f_{j}}=v_{j} \mid U$ for $j \in J$,

(2) $f_{j} \rightarrow f \not \equiv 0(j \rightarrow J)$ uniformly on $U$.

A family $\mathfrak{R}$ of nonnegative divisors on a complex manifold is said to be normal iff every subnet of $\mathfrak{R}$ has a convergent subnet. The following Montel-type theorem is due to Stoll [13, Theorem 2.24, p. 188].

Proposition 12.2. Let $\mathfrak{N}=\left\{\nu_{\lambda}\right\}_{\lambda \in \Lambda}$ be a family of nonnegative divisors on the $n$ dimensional complex manifold $G$. Then $\mathfrak{R}$ is normal iff for each $a \in G$ there exist an open set $U$ in $C^{n}$ and a biholomorphic map $\alpha: U \rightarrow G$ such that

(1) $a \in \alpha(U)$,

(2) $\sup \left\{V_{v_{\lambda} \circ \alpha}(U) \mid \lambda \in \Lambda\right\}<\infty$.

Let $\mathfrak{R}=\left\{v_{\lambda}\right\}_{\lambda \in \Lambda}$ be a family of nonnegative divisors on the complex manifold $G$. If $U$ is an open subset of $G$, we define

$$
\mathfrak{N} \mid U=\left\{v_{\lambda} \mid U\right\}_{\lambda \in \Lambda}
$$

$\mathfrak{N} \mid U$ is a family of nonnegative divisors on $U$. Clearly $\mathfrak{R}$ is normal iff it is locally normal, i.e., for each $a \in G$ there exists an open neighborhood $U$ such that $\mathfrak{R} \mid U$ is normal.

DEFINITION 12.3. Let $\mathfrak{N}$ be a family of nonnegative divisors on the complex manifold $G$. The normality domain of $\mathfrak{R}$ is the largest open set $D \subset G$ such that $\mathfrak{N} \mid D$ is normal.

Finally, we show how a result of Oka [7, Theorem 2, p. 95] and O. Fujita [3, Theorem 1, p. 385] can be obtained from 11.2. 
THEOREM 12.4. Let $\mathfrak{R}=\left\{v_{\lambda}\right\}_{\lambda \in \Lambda}$ be a family of nonnegative divisors on the Stein manifold $G$. Then the normality domain of $\mathfrak{R}$ is a Stein manifold.

Proof. Define $a_{\lambda}=1$ for $\lambda \in \Lambda$, and let $A=\left\{a_{\lambda}\right\}_{\lambda \in \Lambda}$. In view of 12.2, the normality domain of $\mathfrak{R}$ is

$$
\{z \mid z \in G, z \text { is of the first kind w.r.t. } \mathfrak{R} \text { and } A\} .
$$

According to 11.2 , this is a Stein manifold, Q.E.D.

\section{BIBLIOGRAPHY}

1. H. J. Bremermann, "On Oka's theorem for Stein manifolds," in Seminars on analytic functions, Vol. I, pp. 29-35, Institute for Advanced Study, Princeton, N. J., (USAFOSR), 1957.

2. C. Chevalley, Theory of Lie groups, Princeton Univ. Press., Princeton, N. J., 1946.

3. O. Fujita, Sur les familles d'ensembles analytiques, J. Math. Soc. Japan 16 (1964), 379-405.

4. —_, Sur les suites de surfaces analytiques, J. Math. Kyoto Univ. 4 (1965), 627-635.

5. R. C. Gunning and H. Rossi, Analytic functions of several complex variables, PrenticeHall, Englewood Cliffs, N. J., 1965.

6. E. Hille, Analytic function theory, Ginn, Boston, Mass., 1959.

7. K. Oka, Note sur les familles de fonctions analytiques multiformes etc., J. Sci. Hiroshima Univ. A4 (1934) 94-98.

8. - Sur les fonctions analytiques de plusieurs variables $X$-Une mode nouvelle engendrant les domains pseudoconvexes, Japan J. Math. 32 (1962), 1-12.

9. A. Sard, The measure of the critical values of differentiable maps, Bull. Amer. Math. Soc. 48 (1942), 883-890.

10. W. Stoll, Mehrfache Integrale auf komplexen Mannigfaltigkeiten, Math. Z. 57 (1952), 116-154.

11. — Die beiden Hauptsätze der Wertverteilungstheorie bei Funktionen mehrerer komplexer Veränderlichen. (I), Acta Math. 90 (1953), 1-115.

12. - U Uber meromorphe Abbildungen komplexer Räume. II, Math. Ann. 136 (1958), 393-429.

13. - Normal families of non-negative divisors, Math. Z. 84 (1964), 154-218.

UNIVERSITY OF CALIFORNIA,

Riverside, California 